\title{
EPR Investigation Of Mn (II) Probe In Diaquamalonatozinc(II) Single Crystals - Usage In Enrichment Of Dental Cement
}

\author{
S. Deepa* and B. Natarajan ${ }^{\#}$ \\ *Department of Chemistry, Sri Manakula Vinayagar Engineering College, Puducherry - 605 107, India. \\ \#Department of Chemistry, SRM University, Kattankulathur - 603 203, India.
}

\begin{abstract}
Single crystal EPR studies on $\mathrm{Cu}(\mathrm{II})$ and VO(II) doped Diaquamalonatozinc(II) are already studied and published. Inorder to understand the effect of $\mathrm{Mn}(I I)$ in the host lattice Diaquamalonatozinc(II), single crstal EPR studies of Mn(II) doped Diaquamalonatozinc(II) are carried out at room temperature to identify the local structure of the host ion. Angular variation of Mn(II) hyperfine lines in the three orthogonal planes shows the presence of a two sites. The spin-Hamiltonian parameters calculated from the single crystal EPR spectra are: $g x x=1.989$, gyy $=1.999, g z z=2.023 ; A x x=8.72, A y y=9.28 ; A z z=9.34 m T, D x x=-33.36$, Dyy $=-7.25$, $D z z=40.61 \mathrm{mT}$ and $E=13.05 \mathrm{mT}$. The spin-Hamiltonian parameters calculated from the powder EPR spectrum are: $g=1.995, A=9.25 \mathrm{mT}, D=-53.6$. The percentage of covalency obtained is around $8.5 \%$.
\end{abstract}

Keywords: Diamagnetic host lattice, EPR, Mn(II), Single crystal, Two sites.

\section{Introduction}

Diaquamalonatozinc(II) single crystals, first synthesized in 1982 [1], find its application in the enrichment of dental cements. Diaquamalonatozinc(II), hereafter abbreviated as DAMZ, is undertaken to evaluate the binding of $\mathrm{Zn}$ (II) by the malonate ion as a potentially more attractive fortifying agent for the $\mathrm{ZnO}$ /eugenol cements. A similar crystal structure, already found in the literature is diaquamalonatocalcium(II) [2]. Generally polyfunctional carboxylate systems are found to be less sterically constrained in their bridging potential than 2-ethoxybenzoic acid (o-eba) which is also used in strengthening the $\mathrm{ZnO}$ /eugenol materials. This instigates the preparation of the new crystal lattice DAMZ. Moreover, it is quite interesting to probe a paramagnetic ion in the crystal lattice in order to find the crystal field environment. Electron paramagnetic resonance studies are carried out by doping Mn(II) in DAMZ single crystals. EPR studies provide a detail description about the nature of electric field symmetry produced by ligands around the paramagnetic ions. Paramagnetic compounds with divalent metal ion, such as $\mathrm{Mn}$ (II), are of more interest because the $3 \mathrm{~d}$ electron shell responsible for paramagnetism is just half filled with five electrons and the resultant angular momentum is zero. Moreover the ground state of $\mathrm{Mn}$ (II) is ${ }^{6} \mathrm{~S}_{5 / 2}$ and the S-state ions are characterized by long spin lattice relaxation times. This long spin-lattice relaxation time for $\mathrm{Mn}$ (II) ion makes the EPR spectra to be observed at room temperature itself. Its zero field tensor is highly sensitive to the strength and symmetry of the crystal field environments (3-12). Using Mn(II) as spin probe, here we report the EPR study of site symmetry of Mn in DAMZ. Detailed single crystal EPR study on $\mathrm{Mn}$ (II) is found to cover several additional features of interest. For instance, one can study both the forbidden $\left(\Delta \mathrm{M}_{\mathrm{s}}= \pm 1, \Delta \mathrm{M}_{\mathrm{I}}= \pm 1\right.$ or \pm 2$)$ and allowed $\left(\Delta \mathrm{M}_{\mathrm{s}}= \pm 1, \Delta \mathrm{M}_{\mathrm{I}}= \pm\right.$ 0 ) transitions in favorable situations (13-18). Even if the crystal structure is not precisely known, the principle axis can be fixed by the disappearance of the forbidden transitions. Further the deviation of the $g$-values from the free spin value in $\mathrm{d}^{5}$ systems has been the subject of considerable discussion (19-20). In the present case, the impurity ion site encountered is found to be favorable for such discussion.

\subsection{Methodology}

\section{Experimental}

$\left[\mathrm{Zn}\left(\mathrm{C}_{3} \mathrm{H}_{2} \mathrm{O}_{4}\right)\left(\mathrm{H}_{2} \mathrm{O}\right)_{2}\right]$ is prepared as follows: malonic acid $\left(\mathrm{C}_{3} \mathrm{H}_{2} \mathrm{O}_{4}\right)(4.57 \mathrm{~g})$ is dissolved in hot aqueous $\mathrm{MeOH}(1: 1)$ and added to a suspension of $\mathrm{ZnCO}_{3} \cdot 2 \mathrm{ZnO} \cdot 3 \mathrm{H}_{2} \mathrm{O}(14.3 \mathrm{~g})$ in $30 \mathrm{ml}$ of water. The reaction mixture is stirred for 15 minutes and filtered. To the filtrate, a few drops of manganese sulphate is added as the paramagnetic impurity and the reaction mixture is allowed to stand at room temperature for slow evaporation. Crystals are obtained after 6 days.

\subsection{Crystal Structure}

A detailed crystal structure of DAMZ is reported [1]. The unit cell of DAMZ is monoclinic with a $=$ $1.258, \mathrm{~b}=0.741, \mathrm{c}=0.723 \mathrm{~nm} ; \beta=119.0^{\circ}$ and contains four molecules related by the operation of the space group $\mathrm{C} 2 / \mathrm{m}[1]$. The structure comprises non-equivalent zinc ions at $(0,0,0) \&(0,1 / 2,1 / 2)$ with site symmetry $2 / \mathrm{m}$ and bridged by a carboxyl group. A water molecule is coordinated to each metal ion and the central carbon atom of the malonate ion lies on the mirror plane. Each zinc atom is surrounded by a distorted octahedron of six 
water molecules, such that two water molecules are at a distance 0.206 and $0.215 \mathrm{~nm}$ and four oxygen atoms of the two malonato groups are at 0.213 and $0.202 \mathrm{~nm}$ [1]. Single crystal rotations are done along the three mutually perpendicular crystallographic axes, viz., a, b and axis $\mathrm{c}^{*}$. Here, $\mathrm{c}^{*}$ is perpendicular to crystallographic a axis in the ab plane.

\section{Results And Discussion}

As mentioned earlier, single crystal rotations are carried out in the three mutually orthogonal planes, i.e., ab, ac* and bc*. Generally a 30 line pattern is expected for a single $\mathrm{Mn}(\mathrm{II})$ ion.. Manganese ion (Mn(II)) has got a total spin of $5 / 2$ will give just one EPR line, in the absence of any zero-field splitting. The ground state ${ }^{6} \mathrm{~S}_{5 / 2}$ splits into three Kramers' doublets, i.e., $| \pm 5 / 2>,| \pm 3 / 2>$, and $\mid \pm 1 / 2>$, in the absence of any applied magnetic field, with separations of 4D and 2D respectively due to spin-spin interaction. These Kramers' doublets split further by the application of a magnetic field along the symmetry axis with successive levels such as $g \beta B+4 D$, $g \beta B+2 D, g \beta B, g \beta B-2 D, g \beta B-4 D$. These six levels give rise to five fine structure transitions. Each line splits further into a sextet due to the hyperfine structure from the nuclear spin $\mathrm{I}=5 / 2\left({ }^{55} \mathrm{Mn}\right)$. Thus a 30 line pattern is expected per molecule per unit cell.

At an arbitrary orientation, more than 30 line EPR spectrum is characteristic of more than one magnetically distinct Mn(II) sites per unit cell. However from the crystal data, the unit cell contains four molecules per unit. Since Mn(II) is doped into $\mathrm{Zn}$ (II), there is no need for charge compensation. A typical EPR spectrum of $\mathrm{Mn}(\mathrm{II}) / \mathrm{DAMZ}$ in ac* plane of rotation, when the applied magnetic field (B) is parallel to crystallographic a-axis, is given in Figure 1. A close look at the spectrum indicates the presence of more than 30 lines (due to the presence of more than six lines at the centre portion) and the central sextet is relatively higher in intensity than the other four sextets. The expected intensity ratio of the five zero-field transitions in Manganese (II) ion is 5:8:9:8:5. However, the spectrum does not show this intensity ratio because of the presence of other zero-field splitting terms ' $a$ ' and ' $F$ '. Moreover, the second order effects also make the hyperfine lines unequally spaced. Another interesting thing observed here is that the low field and high field transitions only are resolved in most of the orientations, whereas the intermediate transitions show considerable overlap.

This type of overlap indicates the presence of two magnetically inequivalent sites in the lattice, one with large $\mathrm{D}$ value and the other with a small $\mathrm{D}$ value. It is to be recalled here that the number of molecules per unit cell reported from X-ray data is four [1]. Here, it should be noted that two of the four molecules of DAMZ are identical, while the other two are derived from the above pair by a reflection in the crystallographic ac plane. However, as far as the magnetic resonance properties are concerned, all the four sites of $\mathrm{Zn}$ (II) are identical and hence only two sites are expected.

A few EPR spectra at indicated orientations $\left(0^{\circ}, 90^{\circ}, 120^{\circ}\right.$ degrees $)$ in $b c^{*}$ plane at room temperature are shown in Figure 2. The top figure shows the maximum spread, corresponding to the orientation when $\mathrm{B}$ is parallel to crystallographic c-axis; the middle figure corresponds to the orientation, when B is parallel to b-axis and the bottom figure represents the minimum spread of the spectrum in $\mathrm{bc}^{*}$ plane, which roughly corresponds to the magic angle. In all the three planes, the spectra are found to be highly anisotropic and unsymmetrical indicating the non-zero value for the asymmetry parameter E. Isofrequency plots, drawn for all three planes $a c^{*}, b^{*}$ and ab are shown in Figures 3, 4 and 5 respectively. In the ac* plane isofrequency plot, the maximum spread noticed at $\theta=0^{\circ}$ corresponds to $8 \mathrm{D}$, i.e, $2 \mathrm{D}\left(3 \cos ^{2} \theta-1\right)$, to first order, neglecting $\mathrm{E}$ term. This maximum spread is the separation between $|+5 / 2>\leftrightarrow|+3 / 2>$ and $|-5 / 2>\leftrightarrow|-3 / 2\rangle$ transitions. At $\theta=54^{\circ} .47^{\prime}$ the term in the bracket becomes zero i.e., $\left(3 \cos ^{2} \theta-1\right)=0$. A second maximum is observed at $\theta=90^{\circ}$ which corresponds to $4 \mathrm{D}$. This pattern again repeats at $\theta=180^{\circ}$. A similar pattern is noticed in $b c^{*}$ plane also. However, in ab plane of rotation, no crossover of lines occurs and it is clearly visible in Figure 5. If it happens to be invariant in the position of resonance lines, then it can be attributed to the fact that the $g_{x x}=g_{y y}, A_{x x}=A_{y y}$ and $D_{x x}=D_{y y}$. Variations in resonance lines can arise only if there is small variation in the spin Hamiltonian parameters. In the present case, the ab plane does not have the invariant resonance lines. The observed EPR spectra in all the three planes can be described by the spin -Hamiltonian [21]

$$
\begin{aligned}
\mathscr{H}= & \beta \text { B.g.S }+\left[\mathrm{A}_{\mathrm{zz}} \mathrm{S}_{\mathrm{z}} \mathrm{I}_{\mathrm{z}}+\mathrm{A}_{\mathrm{xx}} \mathrm{S}_{\mathrm{x}} \mathrm{I}_{\mathrm{x}}+\mathrm{A}_{\mathrm{xx}} \mathrm{S}_{\mathrm{y}} \mathrm{I}_{\mathrm{y}}\right]+\mathrm{D}\left[\mathrm{Sz}^{2}-1 / 3 \mathrm{~S}(\mathrm{~S}+1)\right] \\
& +\mathrm{E}\left(\mathrm{S}_{\mathrm{x}}^{2}-\mathrm{S}_{\mathrm{y}}^{2}\right)+(\mathrm{a} / 6)\left[\mathrm{S}_{\alpha}^{4}+\mathrm{S}_{\beta}{ }^{4}+\mathrm{S}_{\gamma}{ }^{4}-(1 / 5) \mathrm{S}(\mathrm{S}+1)\left(3 \mathrm{~S}^{2}+3 \mathrm{~S}-1\right)\right] \\
& +(\mathrm{F} / 180)\left[35 \mathrm{~S}_{\mathrm{z}}^{4}-30 \mathrm{~S}(\mathrm{~S}+1) \mathrm{S}_{\mathrm{z}}^{2}+25 \mathrm{~S}_{\mathrm{z}}^{2}-6 \mathrm{~S}(\mathrm{~S}+1)+3 \mathrm{~S}^{2}(\mathrm{~S}+1)^{2}\right]
\end{aligned}
$$

Here, the first term represents electron Zeeman, the second and third terms symbolize the hyperfine interaction and axial components of the zero-field splitting, respectively. The fourth and the fifth term correspond to deviation from axial symmetry and the interaction with cubic crystal field, respectively. The sixth term represents the deviation from cubic symmetry.

As the system contains more than one type of centers, the analysis has been done for only one site, using EPR-NMR program [22]. The spin Hamiltonian parameters obtained from the EPR-NMR program [22] 
are given in Table 1, along with the direction cosines of $\mathrm{g}, \mathrm{A}$ and $\mathrm{D}$ matrices. A few literature results are given in Table 2, for comparison, where the agreement is good between the current values and the reported values. The reported $\mathrm{g}$ values: $\mathrm{g}_{\mathrm{xx}}=1.989 ; \mathrm{g}_{\mathrm{yy}}=1.999$ and $\mathrm{g}_{\mathrm{zz}}=2.023$ show large anisotropy. The deviation from the free spin value (2.0023) has been observed in distorted octahedral systems. A similar observation is known in cases of $\mathrm{Mn}$ (II) doped in tris(ethylenediamine) zinc(II) [23], zinc acetate dihydrate [24] and $\mathrm{Ca}_{2} \mathrm{Ba}(\mathrm{COOH})_{6}$ [25]. The large observed shift of the g-values from the free ion value for $\mathrm{Cu}(\mathrm{II})$ in low symmetry can be well accounted by mixing the excited state into the ground state [26-28]. However, the admixtures of the excited quartet levels into the ground state ${ }^{6} \mathrm{~S}$ by spin-orbit coupling produce only a small negative g-shift. A more significant contribution to leading to the positive g-shift as observed arises due to the electron transfer process through spin-orbit interaction from the ligands to the S-state ion. An estimate of the covalency in the present study is obtained from the hyperfine splitting constant. The large D value may be due to the steric hindrance caused by the malonate rings. In order to understand the location of the paramagnetic ion in the complex as interstitial or substitutional, the direction cosines of metal-oxygen bonds, calculated from the X-ray data of the host lattice, are given in Table 3. None of the direction cosines of spin Hamiltonian matrices of the impurity match with the $\mathrm{Zn}-\mathrm{O}$ bond directions. This suggests that the impurity might have entered the lattice interstitially and not substitutionally (see below). Using the spin Hamiltonian parameters obtained from the EPR-NMR program, the isofrequency plots are simulated and are also given in Figures 3, 4 and 5. In all these figures, the open circles represent the experimental points and the solid lines are calculated values from the EPR-NMR program. A good agreement between the experimental and calculated field positions is found in all the three planes.

\subsection{Relative signs of $D$ and $A$}

Since the magnitude and sign of $\mathrm{D}$ depends on the micro symmetry of a metal ion, it is interesting to determine the sign of $\mathrm{D}$. A positive $\mathrm{D}$ value is related to a tetragonal compression or trigonal elongation. The relative signs of $\mathrm{D}$ and $\mathrm{A}$ manifest themselves by difference in the hyperfine coupling measured from the low field and high field sextets [29]. If D and A are of the same sign, the separations of hyperfine lines will be greater in the low field sextet than the high field sextet and vice versa. The sign of A is invariably negative in all high spin $\mathrm{Mn}$ (II) complexes [30] due to core polarization of inner orbitals of $\mathrm{Mn}$ (II) and assumed to be the same for the present case. In this present study, the separation of the sextets increases towards the high field which infers that the sign of $\mathrm{D}$ is positive. So the positive value of $\mathrm{D}$ and a finite value of $\mathrm{E}$ are taken together may therefore be identified with a compressed octahedron which is orthorhombically distorted. Moreover, the magnitude of the zero field term $\mathrm{D}$, is a function of overlap, covalency and separation of the ${ }^{4} \mathrm{P}$ excited state from the ${ }^{6} \mathrm{~S}$ ground state. Comparatively, a large $\mathrm{D}$ value is obtained, (see table 1 ) indicates the low lying ${ }^{4} \mathrm{P}$ excited state.

\subsection{Covalency of metal-ligand bond}

As mentioned earlier, the covalency of the present study can be estimated from the hyperfine coupling constant. The percentage of covalency of the Mn-O bond is obtained from Matamura's plot. According to Matamura and Simanek et al [31, 32], the covalency of a bond between manganese and its ligands controls the magnitude of the isotropic hyperfine coupling constant A. It is known that hyperfine value decreases with the increasing covalency. An approximate relationship for the covalency $\mathrm{c}$ of a bond between the atoms $\mathrm{p}$ and $\mathrm{q}$ and their electronegative $\chi_{\mathrm{p}}$ and $\chi_{\mathrm{q}}$ is given by the relation

$$
\mathrm{c}=1 / \mathrm{n}\left[1-0.16\left(\chi_{\mathrm{p}}-\chi_{\mathrm{q}}\right)-0.035\left(\chi_{\mathrm{p}}-\chi_{\mathrm{q}}\right)^{2}\right]
$$

where $\mathrm{n}$ is the number of ligands around $\mathrm{Mn}$ (II) ion. The values employed for the calculation are $\chi_{\mathrm{Mn}}=1.4$ and $\chi_{0}=3.5$ [33]. The percentage of covalency obtained is around 8.5\%. The Mn-O bond seems to be almost ionic with only $8.5 \%$ covalency, thus indicating that binding of the metal with ligands is perhaps due to solid state constraint rather chemical affinity.

\subsection{Location of the impurity}

As mentioned above, the paramagnetic impurity does not enter the lattice sustitutionally. Hence, an interstitial position has been calculated. A close look at Figure 6 indicates that a vacant location can be identified, which has $\mathrm{O}(1), \mathrm{O}\left(1\right.$ "'), $\mathrm{O}(4), \mathrm{O}(5), \mathrm{O}\left(5^{\prime \prime}\right.$ ') and $\mathrm{O}(8)$ has possible bonding atoms. The midpoint of opposite pair of oxygen atoms has taken as the possible location for the paramagnetic impurity $\mathrm{Mn}$ (II). By assuming the location for $\mathrm{Mn}$ (II), the various possible $\mathrm{Mn}-\mathrm{O}$ direction cosines have been calculated and are also given in Table 3. A close look at the direction cosines indicates that one of the g-values and A-values matches with the direction cosines of $\mathrm{Mn}-\mathrm{O}(4)$ suggesting that the calculated location of the paramagnetic impurity is acceptable. The deviation between these two direction cosines is around 9 degrees. The figure indicating the location of $\mathrm{Mn}(\mathrm{II})$ is shown pictorially in Figure 6. 


\subsection{Analysis of powder spectrum}

In order to substantiate the single crystal analysis, powder spectrum of the Mn(II)/DAMZ is recorded at room temperature. The room temperature EPR spectrum is given in Figure 7. Here, one can notice only the central six lines corresponding to the intense sextet in the crystal rotations. However, the other lines are not seen due to g-anistropy. This will correspond to the impurity, which does not vary during crystal rotations.

At higher gains (shown at the lower part of the figure), one can see additional lines, corresponding to the impurity, which has angular variation due to the presence of D. Using the higher gain powder EPR spectrum, the spin Hamiltonian parameters have been calculated for the site, which has non-zero D value and are also given in Table 2 . The crystal and powder spectrum values are in good agreement.

\section{Figures And Tables}

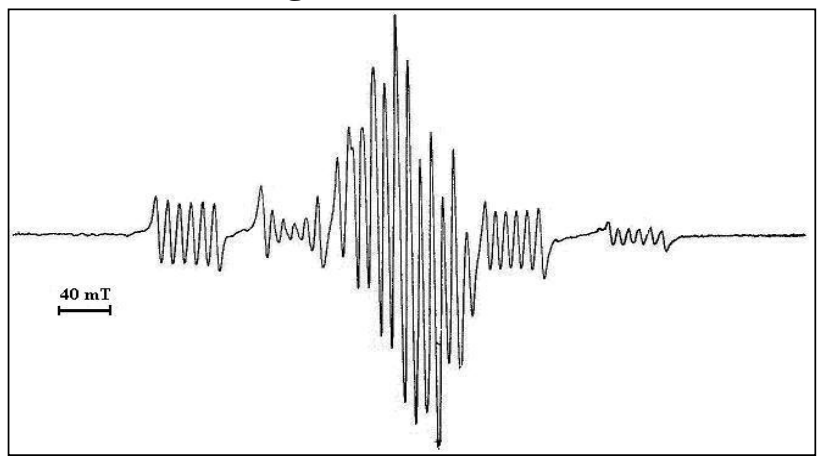

Fig. 1: Single crystal EPR spectrum of Mn(II)/DAMZ at room temperature, when the applied field (B) is parallel to crystallographic a-axis, while rotating the crystal in the ac* plane. Frequency $=9.09248 \mathrm{GHz}$.

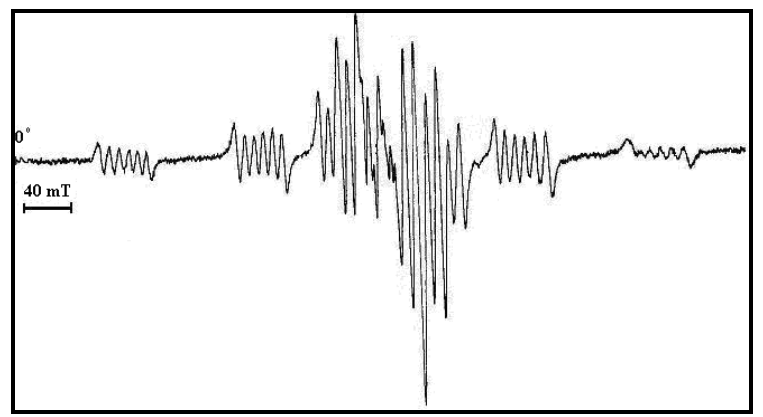

Fig. 2: EPR spectra recorded at room temperature, when magnetic field is parallel $\mathrm{c} *$ plane. Frequency $=$ $9.41905 \mathrm{GHz}$.

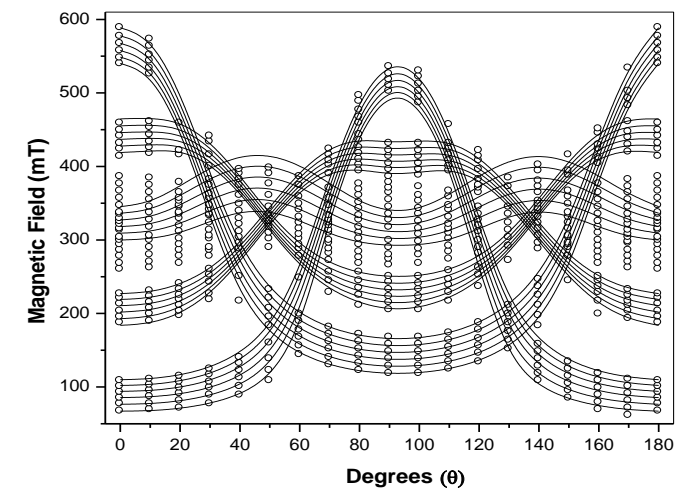

Fig. 3: Rotation pattern for the Mn(II) resonances in the ac* plane of crystal lattice DAMZ. Here $\theta=10^{\circ}$ corresponds to the $\mathrm{c}^{*}$ axis and $\theta=100^{\circ}$ corresponds to the crystallographic a- axis. Here and in Figs 4 and 5, the open circles correspond to the experimental points, whereas the solid lines are calculated values using EPR-

NMR program, with the data given in Table 1 . Frequency $=9.09248 \mathrm{GHz}$. 


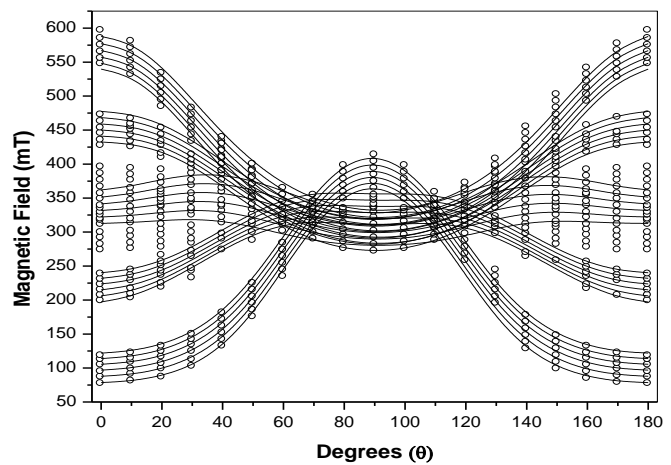

Fig. 4: Rotation pattern for the $\mathrm{Mn}(\mathrm{II})$ resonance in the bc* plane of crystal lattice DAMZ. Here $\theta=0^{\circ}$ corresponds to $\mathrm{c}^{*}$ axis and $\theta=90^{\circ}$ corresponds to the crystallographic b-axis. Frequency $=9.41905 \mathrm{GHz}$.

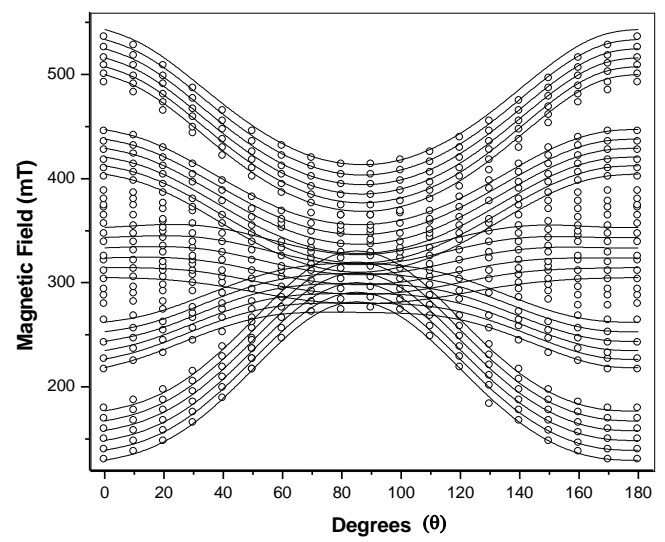

Fig. 5: Rotation pattern for the Mn(II) resonance in the ab plane of crystal lattice DAMZ. Here $\theta=80^{\circ}$ corresponds to the crystallographic $\mathrm{b}$ axis and $\theta=170^{\circ}$ corresponds to the crystallographic a- axis. Frequency $=$ $9.41706 \mathrm{GHz}$.

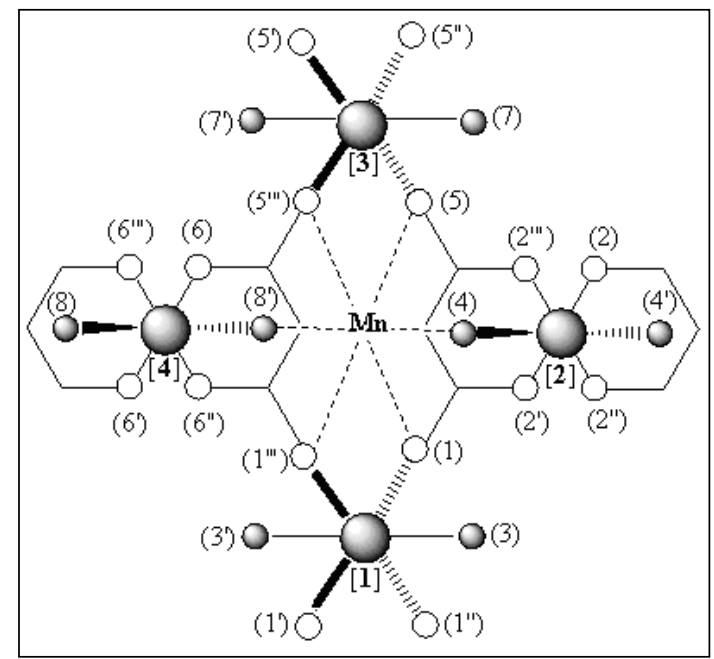

Fig. 6: Schematic representation of the host lattice. The bigger and smaller circles indicates the zinc (numbered in square brackets) and oxygen atoms (shaded circles indicating the oxygen atoms of water molecules and the open circles indicates the malonato oxygens) respectively. The coordinates of the atoms in the host lattice are taken from Noel J. Ray (1). Here the interstitial position of the manganese impurity has been identified and showed. 


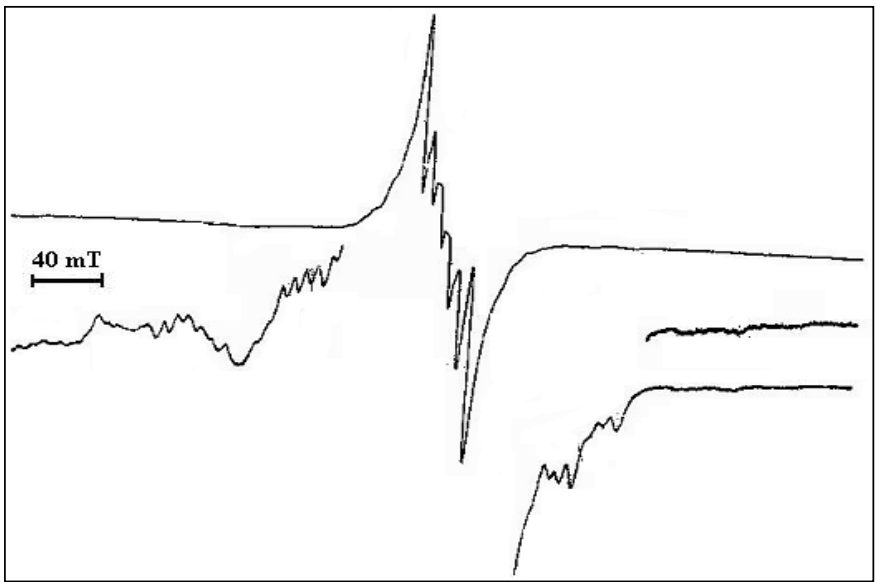

Fig. 7: Polycrystalline EPR spectrum of Mn(II)/DAMZ recorded at room temperature. Frequency $=9.39934$ GHz.

Table 1: The Spin Hamiltonian parameters obtained for $\mathrm{Mn}$ (II) in Diaquamalonatozinc at room temperature and the direction cosines of $\mathrm{Zn}-\mathrm{O}$ bonds, obtained from crystal data [1]. Error in $\mathrm{g}$ is 0.0004 and error in A/D is $0.02 \mathrm{mT}$. See Table 3 also.

\begin{tabular}{|l|l|l|l|}
\hline \multirow{2}{*}{ Principal values } & \multicolumn{2}{|l|}{ Direction Cosines } & \\
\cline { 2 - 4 } & a & b & \\
\hline g values & -0.3569 & -0.3569 & 0.6233 \\
1.989 & 0.1707 & 0.1707 & -0.6886 \\
1.999 & 0.9184 & 0.9184 & 0.3702 \\
2.023 & & & \\
\hline A values (mT) & -0.2764 & 0.569 & 0.7743 \\
8.72 & 0.0185 & 0.8088 & -0.5877 \\
9.28 & 0.9608 & 0.1481 & 0.2342 \\
9.34 & & & \\
\hline D Values (mT) & 0.9882 & 0.0909 & 0.1231 \\
-33.36 & -0.0897 & 0.9958 & -0.015 \\
-7.25 & -0.124 & 0.0038 & 0.9922 \\
40.61 & \multicolumn{2}{|}{} \\
\hline
\end{tabular}

Table 2: The Spin Hamiltonian parameters obtained for Mn(II) in Diaquamalonatozinc at room temperature and some other related systems.

\begin{tabular}{|c|c|c|c|c|c|c|}
\hline \multicolumn{2}{|l|}{ Host lattice } & $\mathbf{g}$ & $\mathbf{A}(\mathrm{mT})$ & $\mathrm{D}(\mathrm{m} T)$ & $\mathbf{E}(\mathbf{m} \mathbf{T})$ & Reference \\
\hline \multicolumn{2}{|l|}{ Zinc maleate Tetrahdrate } & $\begin{array}{l}2.01 \\
1.992 \\
2.035\end{array}$ & $\begin{array}{l}-8.7 \\
-8.7 \\
-8.7\end{array}$ & 36.1 & 6.6 & {$[34]$} \\
\hline \multicolumn{2}{|l|}{ Zinc Acetate dehydrate } & $\begin{array}{l}2.001 \\
2.002 \\
2.001\end{array}$ & $\begin{array}{l}-8.9 \\
-8.9 \\
-8.9\end{array}$ & 24.8 & -2.5 & {$[35]$} \\
\hline \multirow[t]{2}{*}{ Sarcosine Cadmiumbromide } & Site 1 & $\begin{array}{l}1.904 \\
1.939 \\
2.009\end{array}$ & $\begin{array}{l}-8.8 \\
8.9 \\
-9.1\end{array}$ & 46 & -9.8 & \multirow[t]{2}{*}[36]{} \\
\hline & Site 2 & $\begin{array}{l}1.991 \\
1.903 \\
2.012\end{array}$ & $\begin{array}{l}-8.8 \\
8.9 \\
-9.1\end{array}$ & 46 & -9.8 & \\
\hline \multirow[t]{2}{*}{ Diaquamalonato Zinc } & Crystal & $\begin{array}{l}1.989 \\
1.999 \\
2.023 \\
\end{array}$ & $\begin{array}{l}8.72 \\
9.28 \\
9.34 \\
\end{array}$ & $\begin{array}{l}-33.36 \\
-7.25 \\
40.61 \\
\end{array}$ & 13.05 & \multirow[t]{2}{*}{ Present study } \\
\hline & Powder & 1.995 & 9.25 & 53.6 & & \\
\hline
\end{tabular}


Table 3: Direction cosines of Zn-O bonds in DAMZ lattice for sites 1 and II. The oxygen atoms labeled (1) and

(2) are as of malonato ring and the oxygens labeled (3) and (4) are as of water. (For labeling of atoms, see

Figure 6).

\begin{tabular}{|c|c|c|c|c|}
\hline \multirow{2}{*}{\multicolumn{2}{|c|}{ Crystal data }} & \multicolumn{3}{|c|}{ Direction cosines } \\
\hline & & $\mathrm{a}$ & b & $\mathrm{c}^{*}$ \\
\hline \multirow[t]{6}{*}{ Site I } & $\mathrm{Zn}-\mathrm{O}(1)$ & 0.4844 & 0.7271 & 0.491 \\
\hline & $\mathrm{Zn}-\mathrm{O}\left(1^{\prime}\right)$ & -0.4844 & -0.7271 & -0.491 \\
\hline & $\mathrm{Zn}-\mathrm{O}(1 ")$ & 0.4844 & -0.7271 & 0.491 \\
\hline & $\mathrm{Zn}-\mathrm{O}(1 " \prime)$ & -0.4844 & 0.7271 & -0.491 \\
\hline & $\mathrm{Zn}-\mathrm{O}(3)$ & -0.7637 & 0 & 0.6461 \\
\hline & $\mathrm{Zn}-\mathrm{O}\left(3^{\prime}\right)$ & 0.7637 & 0 & -0.6461 \\
\hline \multirow[t]{6}{*}{ Site II } & $\mathrm{Zn}-\mathrm{O}(2)$ & 0.5845 & -0.7104 & -0.3919 \\
\hline & $\mathrm{Zn}-\mathrm{O}\left(2^{\prime}\right)$ & -0.5845 & 0.7104 & 0.3919 \\
\hline & $\mathrm{Zn}-\mathrm{O}(2 ”)$ & 0.5845 & -0.7104 & 0.3919 \\
\hline & $\mathrm{Zn}-\mathrm{O}(2 " ’)$ & -0.5845 & 0.7104 & -0.3919 \\
\hline & $\mathrm{Zn}-\mathrm{O}(4)$ & 0.5068 & 0 & 0.862 \\
\hline & $\mathrm{Zn}-\mathrm{O}\left(4^{\prime}\right)$ & -0.5068 & 0 & -0.862 \\
\hline \multirow[t]{6}{*}{ Interstitial } & $\mathrm{Mn}-\mathrm{O}(1)$ & 0.3954 & -0.8264 & 0.4008 \\
\hline & $\mathrm{Mn}-\mathrm{O}\left(1{ }^{\prime \prime \prime}\right)$ & -0.3954 & -0.8264 & -0.4008 \\
\hline & $\mathrm{Mn}-\mathrm{O}(5)$ & 0.3954 & 0.8264 & 0.4008 \\
\hline & $\mathrm{Mn}-\mathrm{O}(5, ")$ & -0.3954 & 0.8264 & -0.4008 \\
\hline & $\operatorname{Mn}-\mathbf{O}(4)$ & 0.9085 & $\mathbf{0}$ & 0.4177 \\
\hline & $\mathrm{Mn}-\mathrm{O}\left(8^{\prime}\right)$ & -0.0617 & 0.6376 & 0.7691 \\
\hline
\end{tabular}

\section{Conclusions}

Single crystal EPR studies of Mn(II) doped in DAMZ have been carried out at room temperature. Although two sites are found during the angular rotation, only one site has been identified clearly and detailed study is carried out. The other site is difficult to follow and looks like having a very low D value. It is found that the impurity enters in to the crystal lattice interstitially. The presence of E value indicates that the system is of low symmetry. The observed hyperfine splitting constant (A) suggests that the bonding between the central metal ion $(\mathrm{Mn}(\mathrm{II}))$ and the ligand is ionic. Positive sign has been assigned for $\mathrm{D}$ after having a close look at hyperfine structure of the impurity.

\section{Acknowledgement}

The authors express sincere thanks to Pondicherry University and Prof. P. Sambasiva Rao (late) for his constant moral support.

\section{References}

[1] N. J. Ray, Acta Cryst., B 38 (1982) 770.

[2] A. Karipides, A. T. Reed, Acta Cryst. B 36 (1980) 1377.

[3] K. V. Narasimhulu, J. Lakshmana Rao, Physica B 254 (1998) 37.

[4] V. S. X. Anthonisamy, M. Velayutham, R. Murugesan, Physica B 262 (1999) 13.

[5] K. Velavan, R. Venkatesan, P. Sambasiva Rao, J. Phys. Chem. Solids 66 (2005) 876

[6] V. S. X. Anthonisamy, R. Murugesan, Mol. Phys., 93 (1998) 6.

[7] Jesiamma Joseph, P. Sambasiva Rao, Spectrochim. Acta A 52 (1996) 607.

[8] B. Venkataraman, Ind. J. Pure and Applied Phys., 34 (1990) 273.

[9] V. K. Jain, S. K. Yadav, Phys. Rev. B 33 (1986) 5034.

[10] M. A. M. Abuyouseff, A. Escuer, D. Gatteschi, M. A. S. Goher, Inorg. 38 (1993) 5716.

[11] P. Sambasiva Rao, S. Subramanian, Mol. Phys., 54 (1985) 429.

[12] V. K. Jain, G. Lehmann, Phys. Stat. Sol. B 159 (1990) 495.

[13] B. Bleaney, D. J. E. Ingram, Proc. R. Soc. London, Ser, 205 (1951) 336

[14] V. J. Folen, Phys. Rev. A 139 (1965) 1961.

[15] T. Takeda, J. Phys. Soc. Jpn., 23 (1967) 1314.

[16] G. C. Upreti, J. Magn. Reson., 13 (1974) 336

[17] M. Korkmaz, M. Dupont, B. Aktas, J. Phys. Chem. Solids, 45 (1984) 465.

[18] J.M. Baker, B. Bleaney, W. Hayes, Proc. R. Soc. London, Ser. A 247 (1968) 141.

[19] H. Watanabe, J. Phys. Chem. Solids., 25 (1964) 1471.

[20] I. Fidone, K. W. H. Stevens, Proc. Phys. Soc., 73 (1969)116.

[21] A. Abragam, B. Bleaney, "Electron Paramagnetic Resonance of Transition Metal Ions", Clarendon Press, Oxford (1970).

[22] EPR-NMR Program developed by F. Clark, R. S. Dickson, D. B. Fulton, J. Isoya, A. Lent, D. G. Mc Gavin, M. J. Mombourquette, R. H. D. Nuttall, P. S. Rao, H. Rinnerberg, W. C. Tennant, J. A. Weil, 1996, university of Saskatchewan, Saskatoon, Canada, (1996).

[23] R. A. Palmer, M. Chim-Lam Yang, J. C. Hampell, Inorg. Chem. 17 (1978) 2000.

[24] S. K. Misra, M. Kahrizi, Phys. Rev. B 30 (1984) 2920.

[25] S. K. Misra, G. Jerzak, Solid State Commun. 69 (1987) 57.

[26] A. M. F. Benial, V. Ramakrishnan, R. Murugesan, Spectrochim. Acta A 56 (2000) 2775.

[27] C. Shiyamala, R. Venkatesan, P. Sambasiva Rao, Solid State Commun., 128 (2003) 137.

[28] P. Sambasiva Rao, T. M. Rajendiran, R. Venkatesan, N. Madhu, A. V. Chandrasekhar, B. J. Reddy, Y. P. Reddy, R. V. S. S. N. Ravikumar, Spectrochim. Acta A 57 (2001) 2781. 
[29] R. S. Saraswat, G. C. Upreti, J. Phys. (C): Solid State Phys. 10 (1977) 425.

[30] R. Murugesan, A. Thamarai Chelvam, A. Milton Franklin, V. Ramakrishnan, Mol. Phys. 79 (1993) 663.

[31] O. Matumura, J. Phys. Soc. Japan 14 (1959) 108.

[32] E. Simanek, K. A. Muller, J. Phys. Chem. Solids, 31 (1970) 1027.

[33] W. Gordy, W. J. Orville-Thomas, J. Chem. Phys., 24 (1956) 439.

[34] M. Vithal, R. Jaganathan, J. Chem. Soc. Dalton Trans., 983 (1988).

[35] R. Janakiraman, G. C. Upreti, J. Phys. Chem. Solids, 31 (1969) 1419.

[36] D.Pathinettam Pandiyan, C. Muthukrishnan, R. Murugesan, Spectrochim. Acta Part A 58 (2002) 509. 